\title{
PREVALENCE AND DIVERSITY OF UREDINALES FUNGI AT URBAN GREENERIES IN LITHUANIA
}

\author{
Antanina Stankevičienė \\ Kaunas Botanical Garden of Vytautas Magnus University, Lithuania \\ antanina.stankeviciene@vdu.lt
}

\begin{abstract}
Woody plants at urban greeneries in Lithuania are injured by rust fungi of 13 genera, 28 species: Coleosporium pulsatillae, C. tussilaginis, Cronartium flaccidum, C. ribicola, Cumminsiella mirabilissima, Gymnosporangium clavariiforme, G. confusum, G. cornutum, G. sabinae, G. tremelloides, Hyalopsora aspidiotus, M. allii-fragilis, M. allii-populina, M. caprearum, M. epitea, M. laricis-populina, M. populnea, Melampsora ribesii-viminalis, M. salicis-albae, Melampsorella caryophyllacearum, Melampsoridium betulinum, M. carpini, Ochropsora ariae, Phragmidium tuberculatum, Pucciniastrum areolatum, P. symphyti, Uromyces caraganicola, U. pisi-sativi. During 2009 - 2017 Gymnosporangium sabine had the strongest damages (from $0.21 \pm 0.00$ to $2.43 \pm 0.33$ grades) on Pyrus pyreaster, the weakest damages had done G. cornutum on Sorbus intermedia and S. x thuringiaca 'Fastigiata' - $0-1$ \pm 0.58 grades.
\end{abstract}

Key words: woody plants, Uredinales fungi, urban greenery, Lithuania.

\section{Introduction}

Green areas are an important component of urban areas. The basic requirements for greeneries are durability and longevity. In order to achieve this, it is necessary to know the principles of greeneries design and choices of plant assortment (Januškevičius \& Navys, 2012). For formation process, it is important not only to correctly assess plant compositions but also evaluate their ecological, biological properties (Jakovlevas-Mateckis, 2003). Since January $1^{\text {st }}$ 2008 in Lithuania the law on Gardening came into force (2008.01.14, Nr.D1-31). In its program 'On monitoring the status of green areas and green plantations', there were provided tasks and solution tools for data and other information collection on green areas and plantations in the territories assigned to municipalities in order to properly assess, predict and manage their status (Order ..., 2008). It is a part of the work to preserve and develop (introduce) new greeneries in cities, to maintain them as aesthetically, ecologically, historically important elements of the landscape, to form a full-fledged system of green areas (Grikevičius, 2009). These studies accumulate data on a spread of different groups of pathogens.

One of the most commonly occurring plant pathogens are obligately parasitic, host-specialized rust fungi of the order Uredinales in the Basidiomycota. They cause premature defoliation, sprouts deformation, ruin cone seeds (Pucciniastrum areolatum (Fr.) G.H. Otth.), provoke sprouts densification (Melampsorella caryophyllaceaerum (Link) Schroet), etc. Their cognition has a theoretical and practical significance (Minkevičius \& Ignatavičiūtè, 1991). Studies on these fungi in Lithuania began at the beginning of the 19th century and consistently - 20 $0^{\text {th }}$ century (Minkevičius \& Ignatavičiūtè, 1991). During 1937 - 1984 there were described 250 fungi species (Minkevičiaus \& Ignatavičiūtè, 1991; 1993). Subsequently, additional researches were carried out and a monograph "Mycota Lithuania, Uredinales” (1, 2 parts) (Minkevičius \& Ignatavičiūtè, 1991; 1993) prepared.

Evolution life cycles of Uredinales consist of several sporefication stages, which, for the most part, are passed through on plants belonging to two different taxonomic groups (pleomorphism). The complete sporefication cycle consists of five life stages, at which spores are formed: spermagonia (small spore deposits), aecia (aeciospores accumulation, dry, light yellow to orange, wind-dispersed), uredinia (uredioniospores accumulation, dry, orange to rustcolored or purplish, wind-dispersed), telia (teliospores accumulation, vary greatly in color, from dark brown and light brown, not dispersed or are dispersed only short distances), basidiospores (sprout after the rest period from teliospores). Some of rust fungi species pass all five stages - macrocyclic, if telia stage is missed-demicyclic etc. (Minkevičius \& Ignatavičiūtè, 1991). Damp and warm climate intensifies rust fungi development.

Aim of the work: to describe the variety and damage intensity of Uredinales fungi in Lithuanian city urban greeneries.

\section{Materials and Methods}

In order to systematize previous studies of Uredinales fungi in Lithuania, a retrospective analysis of literature sources was performed: using an online access for accumulated literature in Phytopathology group in Kaunas Botanical Garden of Vytautas Magnus University.

In order to determine the prevalence of Uredinales fungi, in 2009 - 2017, the monitoring of plant status in recreational plantations in Lithuania was carried out, taking an example of Alytus and Kaunas cities. These cities have a rich plant variety in their greeneries: woody plants to be observed in Alytus - 4040 (57 
genera, 98 species, 30 cultivars) and in Kaunas, the second largest city of Lithuania - 2441 (52 genera, 79 species, 19 cultivars) (according http://aplinkosauga. alytus.lt/documents/78415/113236/Alytus_\%20 zeldynu\%20stebesena_2017.pdf; www.kaunas. lt/wp-content/uploads/sites/13/2015/04/Kaunas_ stebesena_2017-ATASKAITA.pdf,). The monitoring was carried out annually from July to August. The plant names were described according to M. Griffiths (1997). Rust has damaged 10 genera, 15 species and 2 cultivars plants in recreational urban greeneries.

Uredinales fungi were identified visually, according to disease symptoms and disease agents fungi morphological features, using binocular lupus. Other species fungi were identified by microscoping in accordance to characterizers, according to descriptors: A. Minkevičius \& M. Ignatavičiūtė (1991, 1993), W. A. Sinclair \& H. H. Lyon (2005), L. Orlikowski \& A. Wojdyla (2010). Fungal names are described in accordance with generally accepted (interactive) code Index fungorum (http://www.indexfungorum), the climate conditions in Lithuania are described according to: http://www.meteo.lt/, and optimal condition for fungi development - according to W. A. Sinclair \& H. H. Lyon (2005).
Rust fungi damage intensity was evaluated in grades in a $0-4$ grade system: 0 grades - injured less than $10 \%$ of plant leaves; 1 grade - injured $11-30 \%$ of leaves; 2 grades - injured 31 - 60\%; 3 - injured $61-80 \%$, plant dries noticeably; 4 grades - injured more than $81 \%$ of plant surface, plant dries (Juronis, Snieškienè \& Žeimavičius, 1999).

Disease intensity (average grade of damage) calculated using the formula:

$$
\mathrm{V}=\frac{\Sigma(\mathrm{n} \times \mathrm{b})}{\mathrm{N}}
$$

where $V$ - average grade of damage, $\Sigma(n \cdot b)$ - the sum of multiplications of equally injured (in grades) plant numbers and injury value, $N$ - the number of valuated plants.

\section{Results and Discussion}

Literature analysis ascertains woody plants in Lithuania to be injured by fungi of 13 genera, 28 species, among them fungi of 11 genera, 23 species of macrocyclic development stage and 1 genus, 5 species of demicyclic stage (Table 1)

During the years 2009 - 2017 status monitoring of urban greeneries ascertained the spread and

Uredinales fungi diversity on woody plants in city greeneries in Lithuania, 2009 - 2017

Table 1

\begin{tabular}{|c|c|c|c|}
\hline $\begin{array}{l}\text { Rust fungi species, } \\
\text { developmental cycle: } \\
\text { * macrocyclic, } \\
\text { ** demicyclic }\end{array}$ & $\begin{array}{l}\text { Host plants } \\
\text { (developmental stage: } \\
\text { spermagonia, aecia) }\end{array}$ & $\begin{array}{l}\text { Host plants } \\
\text { (developmental stage: } \\
\text { uredinia, telia) }\end{array}$ & References \\
\hline $\begin{array}{l}\text { Coleosporium pulsatillae } \\
\text { (F. Strauss) Fr.* }\end{array}$ & $\begin{array}{l}\text { Pinus sylvestris, } \\
\text { P. mugo }\end{array}$ & Pulsatilla Mill. & \multirow[t]{3}{*}{ Minkevičius \& Ignatavičiūtè, 1991} \\
\hline \multirow[t]{2}{*}{ C. tussilaginis (Pers.) Lév. * } & Pinus L. & Tussilago L. & \\
\hline & $\begin{array}{l}\text { Pinus sylvestris L., } \\
\text { P. mugo Turra }\end{array}$ & Senecio L. & \\
\hline $\begin{array}{l}\text { Cronartium flaccidum } \\
\text { (Alb. et Schw.) Wint.* }\end{array}$ & Pinus sylvestris L. & $\begin{array}{l}\text { Paeonia L., Asclepias L., } \\
\text { Pedicularis L. ir kt. }\end{array}$ & Minkevičius \& Ignatavičiūtè, 1991 \\
\hline C. ribicola J.C. Fisch. ${ }^{*}$ & Pinus strobus L. & Ribes L. & $\begin{array}{l}\text { Minkevičius \& Ignatavičiūtè, 1991; } \\
\text { Grigaliūnaitė, 2011; Snieškienė \& } \\
\text { Stankevičienė, 2013; Stankevičienė } \\
\text { 2016; Žiogas et al., } 2008\end{array}$ \\
\hline $\begin{array}{l}\text { Cumminsiella mirabilissima } \\
\text { (Peck) Nannf.* }\end{array}$ & $\begin{array}{l}\text { Malonia aquifolium } \\
\text { Nutt. }\end{array}$ & Malonia aquifolium Nutt. & Minkevičius \& Ignatavičiūtè, 1991 \\
\hline $\begin{array}{l}\text { Gymnosporangium } \\
\text { clavariiforme (Wulfen) DC ** }\end{array}$ & $\begin{array}{l}\text { Amelancher Medik., } \\
\text { Crataegus L., Pyrus } \\
\text { L., Sorbus L. }\end{array}$ & Juniperus L. & Minkevičius \& Ignatavičiūtè, 1991 \\
\hline G. confusum Plowr. ** & $\begin{array}{l}\text { Cotoneaster Medik., } \\
\text { Crataegus L., Cydonia } \\
\text { Mill., Pyrus L. }\end{array}$ & Juniperus L. & Grigaliūnaitė, 2011 \\
\hline $\begin{array}{l}\text { G. cornutum Arthur ex F. Kern } \\
(=G . \text { juniperi }) * *\end{array}$ & $\begin{array}{l}\text { Amelanchier Medik., } \\
\text { Sorbus L. }\end{array}$ & Juniperus L. & $\begin{array}{l}\text { Minkevičius \& Ignatavičiūtė, } \\
\text { 1991; Grigaliūnaitè, Meškauskienè, } \\
\text { \& Matelis, 2006; Snieškienė \& } \\
\text { Stankevičienė, } 2013\end{array}$ \\
\hline
\end{tabular}




\begin{tabular}{|c|c|c|c|}
\hline $\begin{array}{c}\text { Rust fungi species, } \\
\text { developmental cycle: } \\
\text { * macrocyclic, } \\
\text { ** demicyclic }\end{array}$ & $\begin{array}{c}\text { Host plants } \\
\text { (developmental stage: } \\
\text { spermagonia, aecia) }\end{array}$ & $\begin{array}{l}\text { Host plants } \\
\text { (developmental stage: } \\
\text { uredinia, telia) }\end{array}$ & References \\
\hline $\begin{array}{l}\text { G. sabine (Dicks.) G. Winter. } \\
* *\end{array}$ & Pyrus L. & Juniperus sabina L. & $\begin{array}{l}\text { Minkevičius \& Ignatavičiūtè, 1991; } \\
\text { Grigaliūnaite, 2011; Grigaliūnaite } \\
\text { \& Matelis, 2014; Nekrošienė, 2006; } \\
\text { Stankevičienė, } 2016\end{array}$ \\
\hline G. tremelloides R. Hartig ** & $\begin{array}{l}\text { Malus Mill., Pyrus L., } \\
\text { Sorbus L. }\end{array}$ & Juniperus L. & Minkevičius \& Ignatavičiūtè, 1991 \\
\hline $\begin{array}{l}\text { Hyalopsora aspidiotus (Peck) } \\
\text { Magnus* }\end{array}$ & $\begin{array}{l}\text { Abies alba Mill. } \\
\text { A. balsamea (L.) Mill. }\end{array}$ & $\begin{array}{l}\text { Gymnocarpium } \\
\text { dryopteris (L.) New. }\end{array}$ & Minkevičius \& Ignatavičiūtè, 1991 \\
\hline $\begin{array}{l}\text { Melampsora allii-fragilis } \\
\text { Kleb.* }\end{array}$ & Allium L. & $\begin{array}{l}\text { Salix fragilis } \mathrm{L} ., \\
\text { S. pentandra } \mathrm{L} .\end{array}$ & Minkevičius \& Ignatavičiūtè, 1991 \\
\hline M. allii-populina Kleb.* & Allium L. & $\begin{array}{l}\text { Populus balsaminifera } \\
\text { L., P.x canadensis } \\
\text { Moench, } P \text {. nigra L. }\end{array}$ & $\begin{array}{l}\text { Minkevičius \& Ignatavičiūtė, 1991; } \\
\text { Stankevičienė \& Snieškienè, } 2012\end{array}$ \\
\hline $\begin{array}{l}\text { M. caprearum Thüm. }{ }^{*} \\
(=\text { M. larici-caprearum) }\end{array}$ & Larix Mill. & Salix L., S. caprea L. & Minkevičius \& Ignatavičiūtè, 1991 \\
\hline $\begin{array}{l}\text { M. epitea Thüm. } * \\
\text { (=M. laricis-epitea })\end{array}$ & Larix Mill. & Salix L., Salix fragilis L. & Minkevičius \& Ignatavičiūtè, 1991 \\
\hline M. laricis-populina Kleb. * & Larix decidua Mill. & $\begin{array}{l}\text { Populus nigra L., } \\
\text { P.x canadensis Moench, } \\
\text { P. suaveolens Fisch. }\end{array}$ & $\begin{array}{l}\text { Minkevičius \& Ignatavičiūtè, 1991; } \\
\text { Grigaliūnaite, 2011; Grigaliūnaitė, } \\
\text { Meškauskienė, \& Matelis, 2007; } \\
\text { Snieškienè \& Stankevičienè, 2013; } \\
\text { Stankevičienė, } 2014\end{array}$ \\
\hline \multirow[t]{2}{*}{$\begin{array}{l}\text { M. populnea } \text { (Pers.) P. Karst.* } \\
\text { (=M. laricis-tremulae; } \\
\text { M. pinitorqua) }\end{array}$} & Larix decidua Mill. & $\begin{array}{l}\text { Populus alba } \mathrm{L} ., \\
\text { P. tremula } \mathrm{L} ., \\
\text { P. balsaminifera } \mathrm{L} .\end{array}$ & Minkevičius \& Ignatavičiūtè, 1991 \\
\hline & $\begin{array}{l}\text { Pinus contorta, } \\
\text { P. mugo Turra, } \\
\text { P. sylvestris } \mathrm{L} \text {. }\end{array}$ & $\begin{array}{l}\text { Populus alba L., } \\
\text { P. tremula L. }\end{array}$ & Žiogas et al., 2008, 2006 \\
\hline M. ribesii-viminalis Kleb.** & Ribes $L$. & Salix viminalis $\mathrm{L}$. & \begin{tabular}{|l|} 
Grigaliūnaitè, Matelis, \& \\
Stackevičienè, 2009 \\
\end{tabular} \\
\hline M. salicis-albae Kleb. * & Allium L. & $\begin{array}{l}\text { Salix alba L., S. fragilis } \\
\text { L. }\end{array}$ & Minkevičius \& Ignatavičiūtè, 1991 \\
\hline $\begin{array}{l}\text { Melampsorella } \\
\text { caryophyllacearum (DC.) J. } \\
\text { Schröt. * }\end{array}$ & Abies Mill. & $\begin{array}{l}\text { Cariophyllaceae: } \\
\text { Agrostema L., Arenaria } \\
\text { L. }\end{array}$ & Minkevičius \& Ignatavičiūtè, 1991 \\
\hline $\begin{array}{l}\text { Melampsoridium betulinum } \\
\text { (Pers.) Kleb.* }\end{array}$ & Larix Mill. & Betula $\mathrm{L}$. & Minkevičius \& Ignatavičiūtè, 1991 \\
\hline M. carpini (Nees) Dietel* & Larix Mill. & Carpinus L., Corylus L. & Minkevičius \& Ignatavičiūtè, 1991 \\
\hline $\begin{array}{l}\text { Ochropsora ariae (Fuckel) } \\
\text { Ramsb.* }\end{array}$ & Anemone L. & $\begin{array}{l}\text { Malus Mill., Pyrus L., } \\
\text { Sorbus L. } \\
\end{array}$ & Minkevičius \& Ignatavičiūtè, 1991 \\
\hline $\begin{array}{l}\text { Phragmidium tuberculatum } \\
\text { J. Müll.* }\end{array}$ & Rosa cult. & Rosa L. & $\begin{array}{l}\text { Minkevičius \& Ignatavičiūtè, 1991; } \\
\text { Grigaliūnaite, } 2011\end{array}$ \\
\hline $\begin{array}{l}\text { Pucciniastrum areolatum } \\
\text { (Fr.) G. H. Otth * } \\
\text { (=Thekopsora areolata) }\end{array}$ & $\begin{array}{l}\text { Picea abies } \\
\text { (L.) H. Karst. }\end{array}$ & $\begin{array}{l}\text { Padus avium Mill., } \\
\text { Prunus L., Cerasus Mill. }\end{array}$ & Minkevičius \& Ignatavičiūtè, 1991 \\
\hline $\begin{array}{l}\text { P. symphyti (DC.) McKenzie } \\
\text { \& Padamsee (=M. symphyti)* }\end{array}$ & Abies alba Mill. & Symphytum L. & Minkevičius \& Ignatavičiūtè, 1991 \\
\hline $\begin{array}{l}\text { Uromyces caraganicola } \\
\text { Henn.* }\end{array}$ & Euphorbia L. & $\begin{array}{l}\text { Caragana arborescens } \\
\text { Fabr. }\end{array}$ & Minkevičius \& Ignatavičiūtè, 1991 \\
\hline $\begin{array}{l}\text { U. pisi-sativi (Pers.) Liro (= } \\
\text { U. laburni)* }\end{array}$ & Euphorbia L. & $\begin{array}{l}\text { Laburnum anagyroides } \\
\text { Med. }\end{array}$ & Minkevičius \& Ignatavičiūtè, 1991 \\
\hline
\end{tabular}

diversity of currently most prevalent rust fungi in urban greeneries (Table 2). During the monitoring of green plantations, it was determined that rust fungi belonging to 5 genera, 11 species in various intensity have injured woody plants of 10 genera, 15 species and 2 cultivars in recreational urban greeneries.

Fungi rust agents mostly common in Lithuania are Gymnosporangium sabinae, G. cornutum 
The diversity and violation intensity of rust fungi in urban greeneries in Lithuania, 2017

\begin{tabular}{|c|c|c|c|c|c|c|c|c|}
\hline \multicolumn{9}{|c|}{ Rust fungi species / Host plants species, number of investigated plants } \\
\hline \multicolumn{9}{|c|}{ Average damage grade / Years } \\
\hline 2009 & 2010 & 2011 & 2012 & 2013 & 2014 & 2015 & 2016 & 2017 \\
\hline \multicolumn{9}{|c|}{ Cronartium flaccidum (Alb. \& Schwein.) G. Winter. / Pinus sylvestris L., 25} \\
\hline & & & & & & $0.01 \pm 0.04$ & 0 & 0 \\
\hline \multicolumn{9}{|c|}{ C. ribicola J. C. Fisch. / Pinus strobus L., 9} \\
\hline & 0 & 0 & $1 \pm 0.36$ & $1.15 \pm 0.14$ & 0 & $1.25 \pm 0.14$ & $1 \pm 0.36$ & \\
\hline \multicolumn{9}{|c|}{ Gymnosporangium clavariiforme Dietel. / Crataegus monogyna Jacq. 68} \\
\hline 0 & 0 & 0 & $0.56 \pm 0.06$ & $0.9 \pm 0.38$ & $2 \pm 0.3$ & $1.18 \pm 0.49$ & $1.18 \pm 0.49$ & $2 \pm 0.17$ \\
\hline \multicolumn{9}{|c|}{ G. confusum Plowr. / Juniperus sabina L., 43} \\
\hline & & & $0.03 \pm 0.1$ & $0.03 \pm 0.1$ & $0.03 \pm 0.1$ & $0.03 \pm 0.1$ & & \\
\hline \multicolumn{9}{|c|}{ G. cornutum Arthur ex F. Kern. / Sorbus intermedia (Ehrh), 51} \\
\hline $0.09 \pm 0.58$ & & 0 & 0 & $1 \pm 0.58$ & $0.01 \pm 0.5$ & $1 \pm 0.58$ & $0.01 \pm 0.5$ & \\
\hline \multicolumn{9}{|c|}{ G. cornutum Arthur ex F. Kern. / Sorbus x thuringiaca 'Fastigiata', 12} \\
\hline 0 & 0 & 0 & 0 & $1 \pm 0.58$ & 0 & $1 \pm 0.58$ & & \\
\hline \multicolumn{9}{|c|}{ G. sabine (Dicks.) G. Winter / Pyrus pyraster Rehder, 7} \\
\hline $0.21 \pm 0.00$ & $2.11 \pm 0.0$ & $0.5 \pm 0.00$ & $1.5 \pm 0.00$ & $1.03 \pm 0.00$ & $1.03 \pm 0.00$ & $2.43 \pm 0.33$ & $1.1 \pm 0.0$ & $1 \pm 0.00$ \\
\hline \multicolumn{9}{|c|}{ Melampsora caprearum Thüm. / Salix alba 'Tristis', 98} \\
\hline & & & $1 \pm 0.00$ & $1 \pm 0.24$ & $0.5 \pm 0.25$ & $1 \pm 0.00$ & & \\
\hline \multicolumn{9}{|c|}{ M. caprearum Thüm. / Salix caprea L., 57} \\
\hline $0.4 \pm 0.01$ & $2 \pm 0.01$ & $2 \pm 0.01$ & $1 \pm 0.01$ & $1.1 \pm 0.01$ & $1.1 \pm 0.01$ & $2 \pm 0.01$ & $0.1 \pm 0.01$ & $0.01 \pm 0.01$ \\
\hline \multicolumn{9}{|c|}{ M. epitea Thüm. / Salix fragilis L., 2} \\
\hline $0.7 \pm 0.37$ & $0.03 \pm 0.37$ & $0.4 \pm 0.37$ & $0.9 \pm 0.37$ & $0 \pm 0.37$ & $1 \pm 0.37$ & $0.09 \pm 0.37$ & $1 \pm 0.37$ & 0 \\
\hline \multicolumn{9}{|c|}{ M. laricis-populina Kleb. / Populus x berolinensis Dipp. 10} \\
\hline 0 & $1.15 \pm 0.17$ & $1.23 \pm 0.57$ & $1 \pm 0.37$ & $1 \pm 0.37$ & $1 \pm 0.65$ & $2.16 \pm 0.17$ & $0.01 \pm 0.37$ & \\
\hline \multicolumn{9}{|c|}{ M. laricis-populina Kleb. / Populus x canadensis Moench, 62} \\
\hline $2 \pm 0.48$ & $2 \pm 0.48$ & 0 & 0 & $1.13 \pm 0.24$ & & $0.01 \pm 0.14$ & & \\
\hline \multicolumn{9}{|c|}{ Melapsoridium betulinum (Pers.) Kleb. / Betula pendula Roth, 508.} \\
\hline $0.01 \pm 0.01$ & $0.01 \pm 0.01$ & $0.01 \pm 0.01$ & $0.01 \pm 0.01$ & 0 & $0.01 \pm 0.01$ & $0,03 \pm 0.01$ & 0 & 0 \\
\hline \multicolumn{9}{|c|}{ M. betulinum (Pers.) Kleb. / Larix decidua Mill., 68} \\
\hline $0.02 \pm 0.01$ & $0.01 \pm 0.01$ & $0.03 \pm 0.01$ & $0.03 \pm 0.01$ & $0.02 \pm 0.14$ & $0.02 \pm 0.01$ & 0 & $0.01 \pm 0.1$ & $0.1 \pm 0.1$ \\
\hline \multicolumn{9}{|c|}{ Phragmidium tuberculatum Jul. Müll. / Rosa canina L., 4} \\
\hline 0 & $2 \pm 0.10$ & $0.85 \pm 0.10$ & $1.14 \pm 0.27$ & $1 \pm 1.12$ & $0.01 \pm 0.29$ & $0.01 \pm 0.04$ & $0.01 \pm 0.29$ & \\
\hline
\end{tabular}

(=G. juniperi) and $G$. clavariiforme. Optimal development for fungi is wet weather in spring and wet, dry weather with temperatures of $18-21^{\circ} \mathrm{C}$ in summers. During the research period, plants of 4 species were injured by various grades (from 0 to $2.43 \pm 0.33$ grades) (Table 2). G. sabine common in Lithuania, during all these years injured Pyrus pyreaster. Fungi of these species hibernate in the tissue of Juniperus commmunis, begins to develop in spring, when humidity occurs, at the temperature of $10-30^{\circ}$ (Labanowski et al., 2001). During the research period Gymnosporangium cornutum ( $=G$. juniperi) had the smallest damage on Sorbus intermedia, $S$. $x$ thuringiaca 'Fastigiata'. In 2010 and 2017, no injuries of this rust were located, and a stronger damage was noticed on S.x thuringiaca 'Fastigiata' in 2013, 2015 (1 \pm 0.58) (Table 2).

Melampsora spp. is a fungus cosmopolitan (Sinclair \& Lyon, 2005). About 27 species of Melampsora genus fungi is being taken into account in Lithuania. They are morphologically similar, and therefore host plants are often regarded for their identification (Minkevičius \& Ignatavičiūtè, 1991). We managed to detect 3 species on plants of 5 species (Table 2). Young (up to 10 years) pine trees at arboretums are often injured by $M$. populnea (=M. pinitorqua). Pinus contorta is often injured but more rarely $P$. sylvestris, P. mugo. Their sprouts are distorted in " $\mathrm{S}$ " shape. Failed to detect this rust, as it is a disease spreading in arboretums, especially if Populus spp., P. tremula is 
in vicinity, part of the development of fungus passes on it.

Cronartium ribicola is widespread in Lithuania on Pinus flexilis James, P. strobus L., P. sibirica (Rupr.) Mayer etc.). Fungus develops most favorably at the end of summer with wet weather, temperatures not exceeding $20^{\circ} \mathrm{C}$.

During the research period $P$. strobus was injured most in 2015 (1.25 \pm 0.14 grades). In order to limit the spread of this dangerous disease, P. flexilis, P. strobus, $P$. sibirica should not be taken to grow near currants (Ribes L.), which are the only interim host plants of this rust. In 2015, on thickened, bumpy branches of P. sylvestris L. was detected Cronartium flaccidum. Paeonia L. plants were detected to be growing nearby, whose leaves have dried up yet in July (uredinia, telia develope on them).

Melampsoridium betulinum spread on Larix decidua aside them grow young Betula pendula. It carries a lot harm in arboretum. In spring on the bottom side of Larix spikes form orange in color aecia and Betula is spread over with aeciospores. In summer, orange urediniospores develop on the bottom side of birch leaves Uredinia developed within $13-14$ days at $12{ }^{\circ} \mathrm{C}$, and they were killed by exposure to $30^{\circ} \mathrm{C}$ for 6 hours. In the hot, dry summer of 2015, this rust was hardly detected.

Disease damaged spikes dry, fall before time (Snieškienè, 2015). During research Melampsoridium betulinum was found on both plant species (Table 2).

Phragmidium tuberculatum had the strongest damage on Rosa canina in 2010 ( $2 \pm 0.10)$. The most favorable conditions to spread are at frequent wet periods and temperatures of $18-21^{\circ} \mathrm{C}$ in summer.

In order to limit the spread of fungus, a proper plant arrangement is required. Plants, which are common hosts of development of fungi - disease agents should be planted away from each other. Fallen leaves in autumn should be collected and together with damaged parts of the tree should be dug deep or burned.

Uredinales fungi develop more intensely under humid and warm weather condition (Sinclair \& Lyon, 2005). As the development stages, development time and optimal condition for development of these fungi differ; therefore, we cannot determine the general conclusion for them all.

\section{Conclusions}

1. Woody plants in Lithuania in urban greeneries are injured by rust fungi of 13 genera, 28 species. The largest variety is of Melampsora genus (8 species): M. allii-fragilis Kleb., M. allii-populina Kleb., M. caprearum Thüm., M. epitea Thüm., M. laricis-populina Kleb., M. populnea (Pers.) P. Karst., M. ribesii-viminalis Kleb., M. salicisalbae Kleb. and Gymnosporangium (5): G. clavariiforme (Wulfen) DC, G. confusum Plowr., G. cornutum Arthur ex F. Kern., G. sabinae (Dicks.) G. Winter., G. tremelloides R. Hartig.

2. According to the data of monitoring during 2009 2017, woody plants in urban greeneries in Lithuania are injured by rust fungi of 5 genera, 11 species. They have injured plants of 10 genera, 15 species and 2 cultivars on various intensity. The strongest damage (from $0.21 \pm 0.00$ to $2.43 \pm 0.33$ grades) Gymnosporangium sabine (Dicks.) G. Winter) was on Pyrus pyreaster Rehder, the weakest influence had Gymnosporangium cornutum Arthur ex F. Kern on Sorbus intermedia (Ehrh) Pers.) and S. $x$ thuringiaca 'Fastigiata' $-0-1 \pm 0.58$ grades.

\section{References}

1. Alytaus miesto želdinių ir želdynų būklès 2017 metais stebèsenos rezultatai (Results of the state monitoring of greeneries and green plantations of Alytus city in 2017). Retrieved March 1, 2018, from: http:// aplinkosauga.alytus.lt/documents/78415/113236/Alytus_\%20zeldynu\%20stebesena_2017.pdf. pp. 50. (in Lithuanian).

2. Griffiths, M. (1997). Index of garden plants. Macmillan.

3. Grigaliūnaitè, B. (2011). Sumedèjusių augalų grybai ir kenkejjai Vilniaus universiteto botanikos sode. (Fungi and Pests of Woody Plants in the Botanical Garden of Vilnius University). Scripta Hortici BotaniciUniversitatis Vytauti Magni. 15, 33-42. (in Lithuanian).

4. Grigaliūnaitè, B., Matelis, A., \& Stankevičienè, E. (2009). Želdinių fitosanitarinè būklè Vilniaus miesto bendruomeniniuose kiemuose. (Phytosanitary State of Woody Greenery in the Vilnius City Community Yards). Formalion of Urban Green Areas. Scientific Articles. 1(6), 41-46. (in Lithuanian).

5. Grigaliūnaitè, B., Meškauskienè, V., \& Matelis, A. (2006). Vilniaus miesto nepagrindinių gatvių želdinių fitosanitariné būklè. (Sanitary Condition of Bystreet Woody Greenery in the Urbanized Territories). Formalion of Urban Green Areas. Scientific Articles. 38-42. (in Lithuanian).

6. Grigaliūnaitè, B., Meškauskienè, V., \& Matelis, A. (2007). Vilnios pakrančių augalų fitosanitarinè būklè. (Phytosanitary State of Plants on the Vilnia Riverside). Formalion of Urban Green Areas '2007: Water and Plants in Landscape. Scientific Articles: vanduo ir augalija kraštovaizdyje. 43-47. (in Lithuanian). 
7. Grigaliūnaitè, B., \& Matelis, A. (2014). Sumedejjusių augalų fitosanitarinè būklè Vilniaus priemiesčio rekreacineje teritorijoje. (Phytosanitary State of Woody in Vilnius Suburb recreational Territory). Optimization of Ornamental and Garden Plant Assortiment, Technologies and Environment. Scientific Articles. 5(10), 40-44. (in Lithuanian).

8. Grikevičius, R. (2009). Rekreacinès paskirties želdynų ir agrarinių teritorijų tvarkymo ir apsaugos teisiniai aspektai ir savivaldybių (Druskininkų, Kupiškio, Utenos) patirtis tvarkant želdynus. (Recreational green areas and agrarian areas management and protection of the legal aspects and the municipal (Druskininkai, Kupiškis, Utena) experience managing green areas). Priemiesčio mišku, rekreaciniu ir agrariniu teritoriju želdynu ir želdinių tvarkymas ir apsauga. Mokslinių straipsnių rinkinys. 5-9. (in Lithuanian).

9. Index fungorum (2018). Retrieved March 1, 2018, from: http://www.indexfungorum.org/names/names. asp.

10. Jakovlevas-Mateckis, K.J. (2003). Miesto kraštovaizdžio architektūra. (Urban Landscape Architecture). Vilnius, Mokslas. (in Lithuanian).

11. Januškevičius, L., \& Navys, E. (2012). Želdynų kūrimo ekologinių principų ir asortimento klausimu. (Problem of Assortment and Ecological Principles of Creation of Greenery). Optimization of Ornamental and Garden Plant Assortiment, Technologies and Environment. Scientific Articles. 3(8), 41-48. (in Lithuanian).

12. Juronis, V., Snieškienè, V., \& Žeimavičius, K. (1999). The principles of lignified introduced Plants condition assesment. In Plant genefund accumulation, evaluation and protection in the botanical gardens. International Scientific Conference, Vilnius, 1-2 July 1999. (pp. 22-23).

13. Climate Monthly reviews. 2017 Lithuanian Hydrometeorological Service under the Ministry of Environment. Retrieved January 11, 2018, from: http:/www.meteo.lt/ http://www.meteo.lt/klim_men_ apzv.php?id=51.

14. Kauno miesto želdinių ir želdynų būklès 2017 metais stebėsenos rezultatai (Monitoring results of the state of greeneries and green plantations in Kaunas city in 2017) Retrieved January 11, 2018, from: http. www.kaunas.lt/wp-content/uploads/sites/13/2015/04/Kaunas_stebesena_2017-ATASKAITA.pdf._ (in Lithuanian).

15. Labanowski, G., Orlikowski, L., Soika, G., \& Wojdyla, A. (2001). Ochrona drzew i krzewow iglastych. (Protection of trees and coniferous shrubs). Krakow.(in Polish).

16. Lietuvos respublikos Aplinkos ministro ìsakymas dèl želdynu ir želdinių būklès stebèsenos programos patvirtinimo $2008 \mathrm{~m}$. sausio $14 \mathrm{~d}$. Nr. D1-31 (Order of the Ministry of Environment of the Republic of Lithuania on the approval of the monitoring plan for green areas and plantations 2008 January 14 No. D1-3). Retrieved December 20, 2017, from: http://www.tic.lt/scripts/sarasas2.dll?Tekstas=1\&Id=1. (in Lithuanian).

17. Minkevičius, A., \& Ignatavičiūtè, M. (1991). Mycota Lithuania, Uredinales (Fungi in Lithuania, Uredinales), 5 (1). Vilnius, Mokslo ir enciklopedijų leidykla. (in Lithuanian).

18. Minkevičius, A., \& Ignatavičiūtè, M.(1993). Mycota Lithuania, Uredinales (Fungi in Lithuania, Uredinales), 5(2). Vilnius, Mokslo ir enciklopedijų leidykla. (in Lithuanian).

19. Nekrošienè, R. (2006). Šermukšnių būklè ir jų asortimento plètros galimybès Klaipėdos miesto gatvių želdiniuose. (State of Mountain Ashes and possibilities of their sortiment expansion for the Klaipeda street's green plantation). Formalion of City Green Places '2006: Street Green Plantation. 80-85. (in Lithuanian).

20. Orlikowski, L., \& Wojdyla, A. (2010). Choroby ozdobnych drzew lisciastych, (Diseases of decorative deciduous trees). Krakow. (in Polish).

21. Sinclair, W.A., \& Lyon, H.H. (2005). Diseases of Trees and Shrubs. Cornel University Press.

22. Snieškienė, V. (2015). Maumedžių ligos Pietvakarių Lietuvos miškuose. (Diseases of Larix in the SouthWestern Lithuania). Scripta Hortici BotaniciUniversitatis Vytauti Magni. 19, 86-93. (in Lithuanian).

23. Snieškienè, V., \& Stankevičienè, A. (2013). Augalų grybinių ligų sukèlèjai Vytauto Didžiojo universiteto Kauno botanikos sode. (Plants Pathogens in Kaunas Botanical Garden). Scripta Hortici BotaniciUniversitatis Vytauti Magni. 17, 165-176. (in Lithuanian).

24. Stankevičienè, A. (2014). Svarbiausios sumedejusių augalų ligos Kauno miesto želdiniuose ir želdynuose. (Most Important Diseases of Woody Plants in Kaunas City Greenery and Plantations) Optimization of Ornamental and Garden Plant Assortiment, Technologies and Environment. Scientific Articles. 5(10), 179184. (in Lithuanian). 
25. Stankevičienè, A. (2016). Rekreacinių želdinių būklès stebėsena Alytaus mieste. (Monitoring of Recreational Green Areas in Alytus City). Optimization of Ornamental and Garden Plant Assortiment, Technologies and Environment. Scientific Articles. 7(12), 96-102. (in Lithuanian).

26. Stankevičienè, A., \& Snieškienè, V. (2012). Alytaus miesto rekreacinių želdynų būklè ir dermè miesto kraštovaizdyje (The State of Alytus City Greenery and its Harmony on City Landscape). Formalion of Urban Green Areas. Scientific Articles, 1(9), 176-184. (in Lithuanian).

27. Žiogas, A., Juronis, V., Šnieškienè, V., \& Gabrilavičius, R.(2006). Pathological Condition of Introduced Conifers in the Forests of South-Western and Western Lithuania. Baltic forestry, 12(2), 234-242.

28. Žiogas, A., Juronis, V., Šnieškienè, V., \& Gabrilavičius, R. (2008). Balkaninès, Bankso, suktaspyglès ir veimutinès pušų fitopatologinè būklè Vakarų ir Pietvakarių Lietuvos miškuose. (Phytopathological condition of Pinus banksiana, P. contorta, P. peuce and P. strobes in West and Soutwest Lithuanian forests Human and Nature Safety 2016. (2), 133-136. (in Lithuanian). 